\title{
Harnessing large deformation and instabilities of soft dielectrics: theory, experiment, and application
}

\author{
Zhao, Xuanhe, xz69@duke.edu, Duke University
}

\begin{abstract}
Widely used as insulators, capacitors, and transducers in daily life, soft dielectrics based on polymers and polymeric gels play important roles in modern electrified society. Owning to their mechanical compliance, soft dielectrics subject to voltages frequently undergo large deformation and mechanical instabilities. The deformation and instabilities can lead to detrimental failures in some applications of soft dielectrics such as polymer capacitors and insulating gels, but can also be rationally harnessed to enable novel functions such as artificial muscle, dynamic surface patterning, and energy harvesting. According to mechanical constraints on soft dielectrics, we classify their deformation and instabilities into three generic modes: (i) thinning and pull-in, (ii) electro-creasing to cratering, and (iii) electro-cavitation. We then provide a systematic understanding of different modes of deformation and instabilities of soft dielectrics by integrating state-of-the-art experimental methods and observations, theoretical models, and applications. Based on the understanding, a systematic set of strategies to prevent or harness the deformation and instabilities of soft dielectrics for diverse applications are discussed.
\end{abstract}

